\title{
Construction of Equiangular Signatures for Synchronous CDMA Systems
}

\author{
Robert W. Heath Jr. \\ Dept. of Elect. and Comp. Engr. \\ The University of Texas at Austin \\ 1 University Station C0803 \\ Austin, TX 78712-1084 USA \\ rheath@ece.utexas.edu
}

\author{
Joel A. Tropp \\ Inst. for Comp. Engr. and Sci. (ICES) \\ The University of Texas at Austin \\ 1 University Station C0200 \\ Austin, TX 78712 \\ jtropp@ices.utexas.edu
}

\author{
Inderjit. S. Dhillon \\ Dept. of Comp. Sci. \\ The University of Texas at Austin \\ 1 University Station C0500 \\ Austin, TX 78712 \\ inderjit@cs.utexas.edu
}

\author{
Thomas Strohmer \\ Dept. of Mathematics \\ University of California, Davis \\ Davis, CA 95616 USA \\ strohmer@math.ucdavis.edu
}

\begin{abstract}
Welch bound equality (WBE) signature sequences maximize the uplink sum capacity in direct-spread synchronous code division multiple access (CDMA) systems. WBE sequences have a nice interference invariance property that typically holds only when the system is fully loaded and the signature set must be redesigned and reassigned as the number of active users changes to maintain this property. An additional equiangular constraint on the signature set, however, maintains interference invariance. Finding such signatures requires imposing equiangular side constraints on an inverse eigenvalue problem. This paper presents an alternating projection algorithm that can design WBE sequences that satisfy equiangular side constraints. The proposed algorithm can be used to find Grassmannian frames as well as equiangular tight frames. Though one projection is onto a closed but non convex set, it is shown that this algorithm converges to a fixed point, and these fixed points are partially characterized.
\end{abstract}

\section{INTRODUCTION}

Signature sequences that maximize the sum capacity in the uplink of direct-spread synchronous code division multiple access (CDMA) systems in Gaussian noise are known to satisfy Welch's bound on the total squared correlation with equality [1]. These sequences, known as Welch bound equality (WBE) signature sequences, are determined by the number of users and the dimensionality of the signature space. They have the interesting interference invariance property in that each signature sees exactly the same interference power. Thus the interference experienced by a user is independent of the signature assigned to that user. Unfortunately, when the number of active users changes, the signatures must generally be recomputed and reassigned to maintain the interference invariance [2].

Recently a class of signatures, known as Grassmannian signatures, were introduced that satisfy interference invariance even when subsets of the available users are active [3]. This signature construction is intimately related to the problem of sphere packing in the Grassmann manifold, in this case onedimensional subspaces (lines), and more specifically to the construction of Grassmannian tight frames [4]. The interference invariance properties comes from the fact that because Grassmannian signatures satisfy Welch's lower bound on the maximum correlation with equality, they are equiangular (the correlation is the same for all distinct signature pairs) and maximally spaced with the smallest possible inner product. The equiangular property provides interference invariance.
Unfortunately, signatures that are both equiangular and maximally spaced are quite rare. Some explicit constructions are available in the articles [3], [4], [5]. Signatures derived from good packings in the Grassman manifold, even the best line packings tabulated by Sloane [6], do not generally satisfy the WBE property when they are not equiangular. In cases where such signatures do not exist, we would be satisfied with a WBE whose constituent signatures are close to equiangular. Recently proposed numerical algorithms for finding WBEs (e.g., [7], [8], [9], [10]), however, do not easily incorporate equiangular side constraints.

In this paper, we present an algorithm for finding Welch bound equality signature sequences that are exactly (or nearly) equiangular. Our approach builds on our recently proposed iterative algorithm for constructing CDMA signature sequences [11], which has also been used to find signatures satisfying peak-to-average ratio constraints [12]. The idea is to alternately solve two matrix nearness problems, one that finds the closest signature set satisfying Welch's bound with equality and the other that finds the nearest set of equiangular signatures. This algorithm is related to a method used by Chu for solving an inverse eigenvalue problem [13]. Our algorithm can also be used to find Grassmannian frames as well as equiangular tight frames. We argue that our algorithm converges to a fixed point, and we claim that the class of fixed points contains the desired sequences. Detailed proofs of these results are deferred due to space constraints [14].

\section{Signature Design Preliminaries}

Consider the uplink of a single cell, short code, synchronous CDMA system with $N$ total signatures and a processing gain $d$. Let $\mathbf{x}_{k}$ denote the $d \times 1$ signature, code, or sequence, of user $k$, normalized as $\left\|\mathbf{x}_{k}\right\|=1$ for $k=1, \ldots, N$. We assume that the maximum number of active users allowed in the system is $N \geq d>1$.

If the signatures $\mathbf{x}_{k}$ form an orthogonal set, the length $d$ determines the allowable number of users. It has been shown that nonorthogonal signature sets where $N>m$ users may be necessary to achieve the full sum-capacity of the synchronous single-cell CDMA channel [1]. These sequences are called Welch bound equality sequences [15] since they satisfy the Welch bound on the total squared correlation with equality. 
WBE signature sequences have a number of nice properties as summarized in [15], [16]. Perhaps the most interesting property is that, using WBE sequences, the interference is uniform across all users [16]. The sum total interference in the system is given by $\sum_{k} \sum_{l \neq k}\left|\left\langle\mathbf{x}_{k}, \mathbf{x}_{l}\right\rangle\right|^{2}-N^{2}$ which for WBE sequences is simply $\frac{N^{2}}{d}-N$. Using WBE sequences, the total interference power experienced by user $k$ is

$I(k)=\sum_{l=1}^{N}\left|\left\langle\mathbf{x}_{k}, \mathbf{x}_{l}\right\rangle\right|^{2}-1=\frac{N-d}{d}$ for $k=1,2, \ldots, N$

and is the same for every user. Thus the SINR performance for any user $k$ is simply

$$
\mathrm{SINR}_{k}=\left[\left(\frac{\sigma_{s}^{2}}{\sigma_{v}^{2}}\right)^{-1}+\left(\frac{d}{N-d}\right)^{-1}\right]^{-1}
$$

and the performance only depends on $N$ and $d$. Unfortunately, interference invariance only occurs when the system is fully loaded [2], [15], i.e. $N$ users are active. The reason is that a WBE set for $N>d$ users almost always ceases to be a WBE set if any $M<d$ sequences are removed from or added to the set [3]. Thus if $\bar{N}<N$ users are active, the whole signature set will need to be recomputed for $(d, \bar{N})$ and the signatures reassigned or additional power control will have to compensate for interference inequality.

\section{Equiangular Signatures FOR CDMA Systems}

An interesting subclass of WBE signature sequences, known as Grassmannian signatures, retains the interference invariance property even when a subset of signatures are active [3]. Grassmannian signatures are constructed from optimal packings of lines on the Grassmann manifold. These signature sequences satisfy two important properties:

1) They are equiangular, i.e.,

$$
\left|\left\langle\mathbf{x}_{k}, \mathbf{x}_{l}\right\rangle\right|=c \quad \text { for all } k, l \text { with } k \neq l
$$

for some constant $c \geq 0$.

2) They are maximally spaced, i.e. $c$ in (3) is as small as possible.

The equiangular property means that every signature is equally "far" from every other signature. This is the origin of the interference invariance property. For example, if $\mathcal{N}$ is the set that indexes the active signatures, then the total interference experienced by any user $k=1,2, \ldots, N$ is

$$
I(k)=\sum_{l \in \mathcal{N} / k}\left|\left\langle\mathbf{x}_{k}, \mathbf{x}_{l}\right\rangle\right|^{2}=c(|\mathcal{N}|-1)
$$

which only depends on the cardinality of $\mathcal{N}$.

The maximally spaced property implies that the signature sequence minimizes the maximum angle between the lines generated by $\mathbf{x}_{k}$ and $\mathbf{x}_{l}$. Let

$$
\rho(N, m):=\max _{k, l, k \neq l}\left|\left\langle\mathbf{x}_{k}, \mathbf{x}_{l}\right\rangle\right|
$$

denote the maximum correlation. Grassmannian signatures achieve the lower bound on the maximum correlation for a line packing given by (see [17] for example)

$$
\rho(N, d) \geq \sqrt{\frac{N-d}{d(N-1)}} .
$$

Further, if equality holds in (5), then the signatures are equiangular, maximally spaced, and form a WBE signature sequence set [4].

In general, it is torturous to find signatures that satisfy (5) with equality. Most of the current research has approached the design problem with algebraic tools. A notable triumph of this type is the construction of Kerdock codes over $\mathbb{Z}_{2}$ and $\mathbb{Z}_{4}$ due to Calderbank et al. [5]. Other explicit constructions are discussed in the articles [4], [3]. In the numerical realm, Sloane has used his Gosset software to produce and study sphere packings in real Grassmannian spaces [6]. Sloane's algorithms have been extended to complex Grassmannian spaces in [18]. We are not aware of any other numerical methods.

Some examples of signatures that achieve the bound in (5) are available in [4] but generally they are hard to find. The reason is that while good line packings have been tabulated for various $d$ and $N$, these packings do not necessarily maintain the equiangular property. On the other hand, some equiangular signature sets do not achieve the maximally spaced property, e.g., it is possible to find five equiangular vectors in $\mathbb{R}^{3}$ but they are not maximally spaced. In both cases, the resulting packing may not satisfy the WBE property enjoyed when equality is satisfied and thus may no longer be capacityoptimal.

When signature sequences are not available that satisfy (5) with equality, it is not possible to simultaneously obtain a signature sequence that is equiangular, maximally spaced, and satisfies Welch's bound on the total squared correlation with equality. Since the equiangular property provides interference invariance, it may be of practical interest to sacrifice the maximally spaced requirement but yet maintain the constraint that the signature sequence forms a WBE sequence to ensure sum-capacity maximization. The objective of this paper is to present an algorithm for finding WBEs that are nearly equiangular.

Let $X=\left[\mathbf{x}_{1}, \mathbf{x}_{2}, \ldots, \mathbf{x}_{N}\right]$ be the signature matrix constructed from the signature set. It can be shown that a necessary and sufficient condition for a signature sequence to satisfy the Welch bound with equality is that the $d$ positive singular values of $\mathbf{S}$ are identical. A matrix with this property is called a tight frame. Our goal, then, is to construct a signature matrix $X$ with the following properties.

i. The matrix is a tight frame: $X X^{*}=\alpha I_{d}$.

ii. Each column has the correct norm: $\left\|\mathbf{x}_{n}\right\|=1$.

iii. The columns are equiangular: $\left|\left\langle\mathbf{x}_{k}, \mathbf{x}_{m}\right\rangle\right|=c$ for all $k \neq m$ and some $c$.

In this paper we present an algorithm that tries to calculate such sequences that we call equiangular tight frames. In the sequel, we summarize the method and its theoretical behavior. 


\section{Alternating Projection Preliminaries}

Our technique is based on an alternating projection between Property (i) and Properties (ii)-(iii). The algorithm attempts to compute a nearby matrix (in terms of the Frobenius norm) that satisfies Properties (i)-(iii).

Since the Gram matrix $X^{*} X$ displays all of the inner products, it is more natural to construct the Gram matrix of an equiangular tight frame than to construct the signature matrix directly. Therefore, our algorithm will alternate between the collection of Hermitian matrices that have the correct spectrum and the collection of Hermitian matrices that have sufficiently small off-diagonal entries.

Define a collection that contains the Gram matrices of all $d \times N \alpha$-tight frames:

$$
\begin{aligned}
& \mathscr{G}_{\alpha} \stackrel{\text { def }}{=}\left\{G \in \mathbb{C}^{N \times N}: G=G^{*}\right. \text { and } \\
& G \text { has eigenvalues }(\underbrace{\alpha, \ldots, \alpha}_{d}, 0, \ldots, 0)\} \text {. }
\end{aligned}
$$

The set $\mathscr{G}_{\alpha}$ is essentially the Grassmannian manifold that consists of $d$-dimensional subspaces of $\mathbb{C}^{N}$ [17]. One may also identify the matrices in $\mathscr{G}_{\alpha}$ as rank- $d$ orthogonal projectors, scaled by $\alpha$.

Theorem 1 shows how to find a matrix in $\mathscr{G}_{\alpha}$ nearest to an arbitrary Hermitian matrix.

Theorem 1: Suppose that $Z$ is an $N \times N$ Hermitian matrix with a unitary factorization $U \wedge U^{*}$, where the entries of $\Lambda$ are arranged in algebraically non-increasing order. Let $U_{d}$ be the $N \times d$ matrix formed from the first $d$ columns of $U$. Then $\alpha U_{d} U_{d}{ }^{*}$ is a matrix in $\mathscr{G}_{\alpha}$ that is closest to $Z$ with respect to the Frobenius norm. This closest matrix is unique if and only if $\lambda_{d}$ strictly exceeds $\lambda_{d+1}$.

Proof: See [14] for details.

Let $\mathscr{H}$ be a closed collection of $N \times N$ Hermitian matrices that satisfy the structural constraint set motivated by the equiangular property:

$$
\begin{aligned}
\mathscr{H}_{\mu} \stackrel{\text { def }}{=}\left\{H \in \mathbb{C}^{N \times N}: H=H^{*},\right. \\
\left.\qquad \operatorname{diag} H=\mathbf{m} 1 \text { and } \max _{m \neq n}\left|h_{m n}\right| \leq \mu\right\} .
\end{aligned}
$$

It may seem more natural to require that the off-diagonal entries have modulus exactly equal to $\mu$, but our experience indicates that the present formulation works better, perhaps because $\mathscr{H}_{\mu}$ is convex.

The following proposition shows how to produce the nearest matrix in $\mathscr{H}_{\mu}$.

Proposition 2: Let $Z$ be an arbitrary matrix. With respect to Frobenius norm, the unique matrix in $\mathscr{H}_{\mu}$ closest to $Z$ has a unit diagonal and off-diagonal entries that satisfy

$$
h_{m n}= \begin{cases}z_{m n} & \text { if }\left|z_{m n}\right| \leq \mu \text { and } \\ \mu \mathrm{e}^{\mathrm{i} \arg z_{m n}} & \text { otherwise. }\end{cases}
$$

We use i to denote the imaginary unit.

Proof: The argument is straightforward.

The objective of alternating minimization is to find a solution to the following question.
Problem 1: Find a matrix in $\mathscr{G}_{\alpha}$ that is minimally distant from $\mathscr{H}$ with respect to a given norm.

If the two sets intersect, any solution to this problem will lie in the intersection. Otherwise, the problem requests a tight frame with unit norm columns whose Gram matrix is "most nearly equiangular." We do not mention the problem of producing a matrix in $\mathscr{H}$ that is nearest to $\mathscr{G}_{\alpha}$ because it is not generally possible to factor a matrix in $\mathscr{H}$ to obtain a frame with dimensions $d \times N$.

\section{Statement of the Algorithm}

Practically, implementing to proposed alternating minimizing involves alternately enforcing the two aforementioned constraint sets until reaching a suitable stopping criterion. Convergence is an issue since the tight frame constraint set is non-convex.

\section{Algorithm 1 (Alternating Projection):}

INPUT:

- An arbitrary matrix $S_{0}$

- The number of iterations $J$

OutPut:

- A signature matrix $X_{J}$

\section{PROCEDURE:}

1) Let $j=1$ and $H=S_{0}^{*} S_{0}$.

2) Find $G_{j}$, the Gram matrix nearest to $H_{j-1}$ in Frobenius norm that has Property (i).

3) Find $H_{j}$, the nearest Gram matrix to $G_{j}$ in Frobenius norm that has Properties (ii) and (iii).

4) Increment $j$. Repeat Steps $2-4$ until $j>J$.

5) Solve for $X_{J}$ by factoring $G_{J}$ using a finite-step method such as [19] for example.

\section{Summary of Convergence Results}

The machinery of point-to-set maps is required to understand the convergence of this algorithm, so we must refer the reader to [14] for details. In this section we shall summarize the convergence results.

\section{A. Basic Convergence Results}

It should be clear that alternating projection never increases the distance between successive iterates. This does not mean that it will locate a point of minimal distance between the constraint sets. It can be shown, however, that it is globally convergent in a weak sense.

Define the distance between a point $M$ and a set $\mathscr{Y}$ via

$$
\operatorname{dist}(M, \mathscr{Y})=\inf _{Y \in \mathscr{Y}}\|Y-M\|_{\mathrm{F}} .
$$

Theorem 3 (Global Convergence of Algorithm): Let $\mathscr{Y}$ and $\mathscr{Z}$ be closed sets, one of which is bounded. Suppose that alternating projection generates a sequence of iterates $\left\{\left(Y_{j}, Z_{j}\right)\right\}$. This sequence has at least one accumulation point.

- Every accumulation point lies in $\mathscr{Y} \times \mathscr{Z}$. 
- Every accumulation point $(\bar{Y}, \bar{Z})$ satisfies

$$
\|\bar{Y}-\bar{Z}\|_{\mathrm{F}}=\lim _{j \rightarrow \infty}\left\|Y_{j}-Z_{j}\right\|_{\mathrm{F}} .
$$

- Every accumulation point $(\bar{Y}, \bar{Z})$ satisfies

$$
\|\bar{Y}-\bar{Z}\|_{\mathrm{F}}=\operatorname{dist}(\bar{Y}, \mathscr{Z})=\operatorname{dist}(\bar{Z}, \mathscr{Y}) \text {. }
$$

For a proof of Theorem 3, see the Appendix in [14]. The convergence of the proposed algorithm is geometric at best [20], [21], [22], [23]. This is the major shortfall of alternating projection methods.

Note that the sequence of iterates may have many accumulation points. Moreover, the last condition does not imply that the accumulation point $(\bar{Y}, \bar{Z})$ is a fixed point of the alternating projection. So what are the potential accumulation points of a sequence of iterates? Since the algorithm never increases the distance between successive iterates, the set of accumulation points includes every pair of matrices in $\mathscr{Y} \times \mathscr{Z}$ that lie at minimal distance from each other.

\section{B. Convergence Results}

Besides the general convergence result, Theorem 3, we also obtain a local convergence result.

Theorem 4: Assume that the alternating projection between $\mathscr{G}_{\alpha}$ and $\mathscr{H}_{\mu}$ generates a sequence of iterates $\left\{\left(G_{j}, H_{j}\right)\right\}$, and suppose that there is an iteration $J$ during which $\left\|G_{J}-H_{J}\right\|_{\mathrm{F}}<N /(d \sqrt{2})$. The sequence of iterates possesses at least one accumulation point, say $(\bar{G}, \bar{H})$.

- The accumulation point lies in $\mathscr{G}_{\alpha} \times \mathscr{H}_{\mu}$.

- The pair $(\bar{G}, \bar{H})$ is a fixed point of the alternating projection. In other words, if we applied the algorithm to $\bar{G}$ or to $\bar{H}$, every iterate would equal $(\bar{G}, \bar{H})$.

- The accumulation point satisfies

$$
\|\bar{G}-\bar{H}\|_{\mathrm{F}}=\lim _{j \rightarrow \infty}\left\|G_{j}-H_{j}\right\|_{\mathrm{F}} .
$$

- The component sequences are asymptotically regular, i.e.

$$
\left\|G_{j+1}-G_{j}\right\|_{\mathrm{F}} \rightarrow 0 \quad \text { and } \quad\left\|H_{j+1}-H_{j}\right\|_{\mathrm{F}} \rightarrow 0 .
$$

- Either the component sequences both converge in norm,

$$
\left\|G_{j}-\bar{G}\right\|_{\mathrm{F}} \rightarrow 0 \text { and }\left\|H_{j}-\bar{H}\right\|_{\mathrm{F}} \rightarrow 0,
$$

or the set of accumulation points forms a continuum.

Proof: See the Appendix in [14].

\section{NUMERICAL EXPERIMENTS}

\section{A. Example Construction}

First, let us illustrate just how significant a difference there

\begin{tabular}{|c|c|c|c|c|c|c|c|c|c|c|c|}
\hline \multirow[b]{2}{*}{$N$} & \multicolumn{5}{|c|}{$d$} & \multirow[b]{2}{*}{$N$} & \multicolumn{5}{|c|}{$d$} \\
\hline & 2 & 3 & 4 & 5 & 6 & & 2 & 3 & 4 & 5 & 6 \\
\hline 3 & $\mathbb{R}$ & $\mathbb{R}$ & .. & .. & .. & 20 & .. & .. & .. & . & . \\
\hline 4 & $\mathbb{C}$ & $\mathbb{R}$ & $\mathbb{R}$ & .. & .. & 21 & .. & .. & .. & $\mathbb{C}$ & . \\
\hline 5 & .. & . & $\mathbb{R}$ & $\mathbb{R}$ & .. & 22 & .. & .. & .. & . & . \\
\hline 6 & .. & $\mathbb{R}$ & . & $\mathbb{R}$ & $\mathbb{R}$ & 23 & .. & .. & .. & . & . \\
\hline 7 & .. & $\mathbb{C}$ & $\mathbb{C}$ & . & $\mathbb{R}$ & 24 & .. & .. & .. & . & . \\
\hline 8 & .. & . & $\mathbb{C}$ & . & . & 25 & .. & .. & .. & $\mathbb{C}$ & . \\
\hline 9 & .. & $\mathbb{C}$ & . & . & $\mathbb{C}$ & 26 & .. & .. & .. & .. & . \\
\hline 10 & .. & .. & . & $\mathbb{R}$ & . & 27 & .. & .. & .. & .. & . \\
\hline 11 & .. & .. & . & $\mathbb{C}$ & $\mathbb{C}$ & 28 & .. & .. & .. & .. & . \\
\hline 12 & .. & .. & . & . & $\mathbb{C}$ & 29 & .. & .. & .. & .. & . \\
\hline 13 & .. & .. & $\mathbb{C}$ & . & . & 30 & .. & .. & .. & .. & . \\
\hline 14 & .. & .. & . & . & . & 31 & .. & .. & .. & .. & $\mathbb{C}$ \\
\hline 15 & .. & .. & . & . & . & 32 & .. & .. & .. & .. & . \\
\hline 16 & .. & .. & $\mathbb{C}$ & . & $\mathbb{R}$ & 33 & .. & .. & .. & .. & . \\
\hline 17 & .. &.. & .. & . & . & 34 & .. & .. & .. & .. & . \\
\hline 18 & .. & .. & .. & . & . & 35 & .. & .. & .. & .. & . \\
\hline 19 & .. & .. & .. & . & . & 36 & .. & .. & .. & .. & $\mathbb{C}$ \\
\hline
\end{tabular}
is between vanilla signature matrices and equiangular signature matrices. Here is the Gram matrix of a six-vector, unit-norm tight frame for $\mathbb{R}^{3}$ :

$\left[\begin{array}{rrrrrr}1.0000 & 0.2414 & -0.6303 & 0.5402 & -0.3564 & -0.3543 \\ 0.2414 & 1.0000 & -0.5575 & -0.4578 & 0.5807 & -0.2902 \\ -0.6303 & -0.5575 & 1.0000 & 0.2947 & 0.3521 & -0.2847 \\ 0.5402 & -0.4578 & 0.2947 & 1.0000 & -0.2392 & -0.5954 \\ -0.3564 & 0.5807 & 0.3521 & -0.2392 & 1.0000 & -0.5955 \\ -0.3543 & -0.2902 & -0.2847 & -0.5954 & -0.5955 & 1.0000\end{array}\right]$.

TABLE I

Equiangular WBE Signature Sets

The notations $\mathbb{R}$ and $\mathbb{C}$ respectively indicate that alternating projection was able to compute a real, or complex, equiangular tight frame. Note that every real, equiangular tight frame is automatically a complex, equiangular tight frame. One period (.) means that no real, equiangular tight frame exists, and two periods (..) mean that no equiangular tight frame exists at all.

Notice that the inner-products between vectors are quite disparate, ranging in magnitude between 0.2392 and 0.6303 . These inner products correspond to acute angles of $76.2^{\circ}$ and $50.9^{\circ}$. In fact, this tight frame is pretty tame; the largest inner products in a unit-norm tight frame can be arbitrarily close to one ${ }^{1}$. The Gram matrix of a six-vector, equiangular tight frame for $\mathbb{R}^{3}$ looks quite different:

$\left[\begin{array}{rrrrrr}1.0000 & 0.4472 & -0.4472 & 0.4472 & -0.4472 & -0.4472 \\ 0.4472 & 1.0000 & -0.4472 & -0.4472 & 0.4472 & -0.4472 \\ -0.4472 & -0.4472 & 1.0000 & 0.4472 & 0.4472 & -0.4472 \\ 0.4472 & -0.4472 & 0.4472 & 1.0000 & -0.4472 & -0.4472 \\ -0.4472 & 0.4472 & 0.4472 & -0.4472 & 1.0000 & -0.4472 \\ -0.4472 & -0.4472 & -0.4472 & -0.4472 & -0.4472 & 1.0000\end{array}\right]$

Every pair of vectors meets at an acute angle of $63.4^{\circ}$. The vectors in this frame can be interpreted as the diagonals of an icosahedron [17].

\section{B. Summary of Basic Constructions}

We have used alternating projection to compute equiangular tight frames, both real and complex, in dimensions two through six. The algorithm performed poorly when initialized with random vectors, which led us to adopt a more sophisticated approach. We begin with many random vectors and winnow this collection down by repeatedly removing whatever vector has the largest inner product against another vector. It is fast and easy to design starting points in this manner, yet the results are impressive. These calculations are summarized in Table I. Alternating projection can locate every real, equiangular tight frame signature matrix in dimensions two through six; algebraic considerations eliminate all the

\footnotetext{
${ }^{1}$ To see this, consider a tight frame that contains two copies of an orthonormal basis, where one copy is rotated away from the other by an arbitrarily small angle.
} 


\begin{tabular}{|c|ccccc|}
\hline $\mathrm{d}$ & 4 & 8 & 16 & 32 & 64 \\
$\mathrm{~N}$ & 5 & 9 & 18 & 36 & 70 \\
\hline Minimum Cor. & 0.2500 & 0.1250 & 0.0021 & 0.0006 & 0.0000 \\
Average Cor. & 0.2500 & 0.1250 & 0.0765 & 0.0516 & 0.0326 \\
Std. Dev. Cor. & 0.0000 & 0.0000 & 0.0429 & 0.0301 & 0.0212 \\
Max Cor. & 0.2500 & 0.1250 & 0.1250 & 0.0966 & 0.0607 \\
Max Cor. Packing & 0.2500 & 0.1250 & 0.0911 & 0.0674 & 0.0427 \\
Max Cor. Bound & 0.2500 & 0.1250 & 0.0857 & 0.0598 & 0.0369 \\
\hline
\end{tabular}

TABLE II

\section{NeAR-EQuiangular WBE Signature Sets}

Summary of the correlation behavior of specific WBE sequences resulting from the proposed algorithm. The last three lines compare the maximum correlation of the candidate near-equiangular WBE with the maximum correlation of the best line packing found for $(d, N)$ without the tight frame constraint and the lower bound on the maximum correlation (5).

remaining values of $N$ [4]. In the complex case, the algorithm was able to compute every equiangular tight frame that we know of. Unfortunately, no one has yet developed necessary conditions on the existence of complex, equiangular tight frames aside from the upper bound, $N \leq d^{2}$, and so we have been unable to rule out the existence of other ensembles.

\section{Overloaded System Example}

We have also constructed some WBEs in dimensions of $d=2^{k}$ for $k=2,3, \ldots, 6$ and an overload factor of ten percent. The results of this construction are illustrated in Table II. Constructions $(4,5)$ and $(8,9)$ are exact equiangular tight frames (corresponding to the simplex). In the other cases, the WBEs are only nearly equiangular. Because of the tight frame constraint, the maximum correlation is somewhat higher than that of the best line packing for those combinations (without the tight frame constraint), and is larger than the lower bound. The standard deviation of the correlation between two signatures provides a measure of "equiangularity." Lower values indicate the signatures are more equiangular. In the proposed examples, there is some variability especially for larger dimensions. This is because $N$ is not much bigger than $d$ thus there are fewer degrees of freedom to enforce the equiangular property. For $d=64$ and $N=128$, though, a construction exists that is equiangular and maximally spaced [3].

\section{CONCLUSION}

We have proposed an alternating minimization that is capable of finding optimal CDMA signature sequences that satisfy equiangular side constraints and discussed convergence of the algorithm. This algorithm can also be used to solve for unconstrained optimal CDMA signature sequences, sequences with peak-to-average power ratio side constraints [12], and spectrum constraints. A major issue with the proposed algorithm is that the resulting sequences are generally complex valued and this may lead to implementation challenges. Incorporating binary or finite alphabet constraints on the signatures is an interesting topic for future research.

\section{REFERENCES}

[1] M. Rupf and J. L. Massey, "Optimum sequence multisets for synchronous code-division multiple-access channels," IEEE Trans. Inform. Theory, vol. 40, no. 4, pp. 1261-1266, July 1994.

[2] V. Kravcenko, H. Boche, F. Fitzek, and A. Wolisz, "No need for signaling: Investigation of capacity and quality of service for multi-code CDMA systems usign the wbe ++ approach," in Proc. 4th Int. Workshop on Mob. and Wireless Commun. Networks, 2002, pp. 110-114.

[3] R. W. Heath Jr., T. Strohmer, and A. J. Paulraj, "Grassmanian signatures for CDMA systems," in Proc. of IEEE Global Telecommunications Conf., San Francisco, CA, Dec. 2003.

[4] T. Strohmer and R. W. Heath Jr., "Grassmannian frames with applications to coding and communication," Appl. Comp. Harmonic Anal., vol. 14, no. 3, pp. 257-275, May 2003.

[5] A. R. Calderbank, P. J. Cameron, W. M. Kantor, and J. J. Seidel, " $\mathbb{Z}_{4}$ Kerdock codes, orthogonal spreads and extremal Euclidean line sets," Proc. London Math. Soc., vol. 75, no. 2, pp. 436-480, 1997.

[6] N. J. A. Sloane, "Packing planes in four dimensions and other mysteries," in Proceedings of the Conference on Algebraic Combinatorics and Related Topics, Yamagata, Japan, Nov. 1997.

[7] C. Rose, "CDMA codeword optimization: interference avoidance and class warfare," IEEE Trans. Inform. Theory, vol. 47, no. 6, pp. 23682382, Sept. 2001.

[8] S. Ulukus and R. D. Yates, "Iterative construction of optimum signature sequence sets in synchronous CDMA systems," IEEE Trans. Inform. Theory, vol. 47, no. 5, pp. 1989-1998, 2001.

[9] C. Rose, S. Ulukus, and R. D. Yates, "Wireless systems and interference avoidance," IEEE Trans. Wireless Comm., vol. 1, no. 3, pp. 415-428, Jul. 2002.

[10] P. Anigstein and V. Anantharam, "Ensuring convergence of the MMSE iteration for interference avoidance to the global optimum," IEEE Trans. Inform. Th., vol. 49, no. 4, pp. 873-885, Apr. 2003.

[11] J. A. Tropp, R. W. Heath Jr., and T. Strohmer, "Optimal CDMA signature sequences, inverse eigenvalue problems and alternating projection," in Proceedings of the 2003 IEEE International Symposium on Information Theory, Yokohama, July 2003, p. 407.

[12] J. A. Tropp, I. S. Dhillon, R. W. Heath Jr., and T. Strohmer, "CDMA signature sequences with low peak-to-average-power ratio via alternating projection," in Proceedings of the 37th Annual Asilomar Conference on Signals, Systems and Computers, Monterrey, Nov. 2003.

[13] M. T. Chu, "Constructing a Hermitian matrix from its diagonal entries and eigenvalues," SIAM J. Matrix Anal. Appl., vol. 16, no. 1, pp. 207217, Jan. 1995.

[14] J. A. Tropp, I. Dhillon, R. W. Heath Jr., and T. Strohmer, "Designing structured tight frames via an alternating projection method," Dec. 2003, the University of Texas at Austin, ICES Report 03-50, December 2003, also submitted to the IEEE Trans. Inform. Theory.

[15] D. V. Sarwate, "Meeting the Welch Bound with equality," in Sequences and their Applications. London: Springer, 1998, pp. 79-102.

[16] J. L. Massey and T. Mittelholzer, "Welch's bound and sequence sets for code division multiple-access systems," in Sequences II: Methods in Communication, Security, and Computer Science. New York: SpringerVerlag, 1993, pp. 63-78.

[17] J. H. Conway, R. H. Hardin, and N. J. A. Sloane, "Packing lines, planes, etc.: Packings in Grassmannian spaces," Experimental Math., vol. 5, no. 2, pp. 139-159, 1996.

[18] D. Agrawal, T. J. Richardson, and R. L. Urbanke, "Multiple-antenna signal constellations for fading channels," IEEE Trans. Inform. Theory, vol. 47, no. 6, pp. 2618-2626, Sept. 2001.

[19] N. N. Chan and K.-H. Li, "Diagonal elements and eigenvalues of a real symmetric matrix," J. Math. Anal. Appl., vol. 91, pp. 562-566, 1983.

[20] N. Aronszajn, Introduction to the Theory of Hilbert Spaces, Vol. I. Stillwater, OK: Research Foundation of Oklahoma A \& M College, 1950.

[21] S. Kayalar and H. Weinert, "Error bounds for the method of alternating projections," Math. Control Signal Systems, pp. 43-59, 1988.

[22] J. de Leeuw, Information Systems and Data Analysis. Berlin: Springer, 1994, ch. Block relaxation algorithms in statistics, pp. 308-325.

[23] F. Deutsch, Best Approximation in Inner-Product Spaces. New York: Springer-Verlag, 2001. 